\title{
Clima Organizacional en una Dirección de Administración de Salud Municipal
}

\author{
ORGANIZATIONAL CLIMATE IN A MUNICIPAL HEALTH ADMINISTRATION OFFICE
}

\author{
Alexis González-Burboa', Camilo Manriquez², Macarena Venegas ${ }^{3}$ \\ 1. Matrón. MBA. Colaborador Académico. Departamento de Obstetricia y Puericultura. Facultad de Medicina. Universidad de Concepción. Chile. \\ 2. Licenciado en Estadistica. Departamento de Obstetricia y Puericultura. Facultad de Medicina. Universidad de Concepción. Chile. \\ 3. Ingeniera Comercial. MBA. Gestora Logística. Dirección de Administración de Salud Municipalidad de Concepción. Chile.
}

\section{RESUMEN}

Justificación: Debido al impacto del clima organizacional en el desempeño de las organizaciones, es vital su estudio en aquellas encargadas de la salud de la población. Objetivo: Explorar las percepciones que poseen los trabajadores de la Dirección de Administración de Salud (DAS) de Concepción. Método: Estudio cuantitativo, descriptivo de corte transversal. El universo fue la totalidad de los trabajadores que se encontraban vinculados a la Administración Central de la DAS de Concepción durante el período comprendido entre el 9 y el 13 de julio del año 2012. Se incluyeron en la muestra todos los trabajadores con contrato indefinido que poseían más de dos años de antigüedad $(n=34)$. Se excluyeron los funcionarios con contrato a plazo fijo $(\mathrm{n}=6)$. Los datos fueron recolectados a través de un instrumento adaptado del Organizational Climate Questionarie de Litwin y Stringer. Para el análisis se utilizó el software estadístico InfoStat, versión 2014. Resultados: De los encuestados, un 57,6\% ( $n=19)$ eran mujeres y un 48,9\% $(\mathrm{n}=16)$ pertenecía al área de Administración y Finanzas. El promedio general más bajo fue asignado a la dimensión de recompensa $(2,21 \pm 0,52)$ y el más alto a la dimensión normas $(2,90 \pm 0,44)$. Al estratificar por sexo no se observaron diferencias significativas. Conclusiones: En la organización estudiada se encontró un buen clima organizacional. Existieron similitudes en la valoración del clima entre hombres y mujeres, coincidiendo ambos que el ámbito más débil era el de recompensa.

(González-Burboa A, Manríquez C, Venegas M, 2014. Clima Organizacional en una Dirección de Administración de Salud Municipal. Cienc Trab. Sep-Dic; 16 [51]: 152-157).

Palabras clave: CLIMA ORGANIZACIONAL, MUNICIPAL, SALUD.

\section{ABSTRACT}

Justification: Due to impact of the organizational climate in the organizations performances, is vital to study those in charge of population health. Objective: To explore workers perceptions from Health Administration Office (DAS) of Concepción. Method: Quantitative, descriptive cross-sectional study. The universe was all the workers who were linked to the central administration of the DAS in Concepcion during the period from 9 to 13 July 2012. All permanent contracted employees with more than two years of service $(n=34)$ were included in the sample. Staff with fixed-term contract $(n=6)$ were excluded. Data were collected using an instrument adapted from the Organizational Climate Questionnaire of Litwin and Stringer. Results: Among the respondents, 57.6\% $(n=19)$ were women and $48.9 \%(n=16)$ belonged to the Financial Area: For analysis InfoStat statistical software, version 2014 was used. The lowest overall average was assigned to the dimension of reward $(2.21 \pm 0.52)$ and the highest to the dimension of Rules $(2.90 \pm 0.44)$. When stratified by sex, no significant differences were observed. Conclusions: In the studied organization, a good organizational climate was found. There were similarities in the assessment of climate between men and women, both agreeing that the weakest area was Reward.

Key words: ORGANIZATIONAL CLIMATE, MUNICIPAL, HEALTH.

\section{INTRODUCCIÓN}

La interacción de los recursos humanos con sus organizaciones origina un fenómeno denominado clima organizacional, el cual puede definirse como la percepción que poseen quienes pertenecen a una organización en relación a sus características y

\section{Correspondencia / Correspondence:}

Alexis González-Burboa

Departamento de Obstetricia y Puericultura. Facultad de Medicina, Universidad de Concepción

Chacabuco esquina Janequeo s/n. Casilla 160-C. Concepción, Chile Tel.: +560412204926

e-mail: alexisgonzalez@udec.cl

Recibido: 03 de Septiembre de 2014 / Aceptado: 20 de Octubre 2014 ambiente interno de la misma. ${ }^{1}$ También se puede definir como las percepciones compartidas y el significado ligado a las politicas, prácticas y procedimientos que los miembros de una organización experimentan. ${ }^{2}$ El clima organizacional es considerado un filtro por el cual pasan los fenómenos objetivos de una organización, los cuales al ser percibidos por los individuos que la integran generan una percepción que influye sobre sus motivaciones y comportamientos. ${ }^{3}$

El clima organizacional ha despertado el especial interés de muchos investigadores desde el pasado siglo ${ }^{2,4}$, principalmente al analizar la influencia que los cambios tecnológicos, económicos y sociales han producido en las organizaciones modernas. Su estudio es de vital importancia a causa del enorme impacto que tiene sobre el comportamiento de los miembros de una organización ${ }^{1,5}$, constituyendo una especie de reflejo de la vida de la organización y las condiciones en las cuales los trabajadores se desenvuelven en el ámbito laboral. ${ }^{3}$ 
Históricamente, el estudio del clima organizacional dominó la investigación temprana sobre el medio ambiente organizacional humano en las décadas del 60 y 70 . Se plantea que la investigación cuantitativa seria acerca del clima organizacional comenzó alrededor de 1970, caracterizándose en sus comienzos por generar acuerdos para definirlo, al casi no contarse con orientaciones conceptuales, y considerando escasamente el término “organizacional". A comienzos de la década del 80 la investigación de esta temática siguió las influencias metodológicas características de la psicología industrial de la época, sin embargo, cayó a un segundo plano desplazada por el auge en la investigación de la cultura organizacional que pareció captar mejor la riqueza del medio ambiente organizacional que la investigación del clima. Sin embargo, a través de la década del 90 nuevamente se produjo un cambio en el foco de interés que, como consecuencia, ha reposicionado la investigación del clima organizacional en los años recientes. ${ }^{6}$

La investigación aplicada al clima organizacional posee una abundante producción en la literatura anglosajona, destacando el aporte realizado al ámbito de las organizaciones vinculadas a la gestión de cuidados de salud. Se han realizado investigaciones que han estudiado la influencia del clima organizacional sobre estrategias de intervención implementadas en cuidados primarios de salud ${ }^{7}$, la aplicabilidad de instrumentos para trabajar con los constructos organizacionales en los escenarios de cuidados de la salud $^{8}$, la influencia que el clima ejerce sobre el desempeño de los trabajadores facilitando la adopción de estrategias para detectar e intervenir el uso de drogas ${ }^{9}$, las implicaciones que el clima de las organizaciones tiene con la práctica de cuidados de enfermería en el escenario de los cuidados primarios ${ }^{10}$, la validación de instrumentos para evaluar el clima organizacional con el fin de mejorar la práctica de cuidados de enfermería en instituciones relacionadas a los cuidados primarios de salud ${ }^{11}$, la relación entre las condiciones de trabajo y el bienestar y salud laboral de los profesionales de la salud del área de la enfermería ${ }^{12}$, el estudio de los factores que impactan en la instauración de un clima de calidad $^{13}$, entre otros.

En el contexto latinoamericano, se han encontrado trabajos que han estudiado el clima organizacional con la finalidad de aportar a la mejora de la dirección de establecimientos y servicios de salud $^{14}$, han estudiado su importancia en la gestión del cambio en sistemas organizacionales ${ }^{15}$, han estudiado cómo el clima afecta el desempeño en unidades clínicas especializadas, como es el caso de la unidades de hemodiálisis ${ }^{16}$, han estudiado cómo el clima constituye un factor que influye en el desgaste de los profesionales que trabajan en hospitales ${ }^{17}$, han estudiado el impacto del clima en la salud mental de los profesionales que trabajan en el poder judicial. ${ }^{18}$

Específicamente, en el contexto chileno se ha estudiado como una variable que refleja satisfacción laboral en profesionales de la salud ${ }^{19}$, sindrome de Burnout ${ }^{20}$ y liderazgo. ${ }^{21}$ Además, se han realizado validaciones de instrumentos para medir clima organizacional en profesionales de la salud. ${ }^{22}$ En el ámbito de la atención primaria no existen trabajos que evalúen el clima dentro de las organizaciones administradoras de la salud municipal, entendiendo que esta es una variable que afecta el desempeño de los trabajadores y, por tanto, repercute sobre el soporte logístico y administrativo que otorgan a los centros asistenciales de su dependencia.

El objetivo de esta investigación fue explorar las percepciones que poseen los trabajadores de la Dirección de Administración de Salud (DAS) de Concepción, una organización pública dependiente administrativamente de la Municipalidad de Concepción y dirigida técnicamente por los lineamientos del Servicio de Salud Concepción, respecto de su clima organizacional. La DAS de Concepción posee una Administración Central, seis Centros de Salud Familiar y un Centro de Referencia de derivación comunal que atienden a una población aproximada de 120 mil usuarios de la red pública de salud. Específicamente, este estudio buscó identificar aquellas dimensiones del clima organizacional que representasen fortalezas a ser valoradas por la institución, y debilidades a ser trabajadas para su mejora.

\section{MATERIAL Y MÉTODO}

Estudio cuantitativo, descriptivo de corte transversal. El universo de la muestra estuvo constituido por la totalidad de los trabajadores que se encontraban vinculados a la Administración Central de la DAS de Concepción durante el período comprendido entre el 9 y el 13 de julio del año 2012. Mediante un muestreo no probabilístico se incluyeron todos los trabajadores con contrato indefinido que poseían más de dos años de antigüedad $(n=34)$. Se excluyeron del estudio los funcionarios con contrato a plazo fijo $(n=6)$ quienes, en todos los casos, tenían una permanencia en la institución inferior a los 6 meses. Del total de trabajadores que cumplían los criterios de inclusión, se encuestó a 33 funcionarios; uno no participó por encontrarse con licencia médica durante el período de estudio. En cuanto a la distribución de la muestra por área de trabajo, la mayor parte de los trabajadores pertenecía al área de Administración y Finanzas $(n=19)$, situándose el resto en las áreas de Programas y Proyectos $(n=9)$ y de Recursos Humanos ( $\mathrm{n}=5)$.

Para la recolección de los datos se utilizó un instrumento adaptado del Organizational Climate Questionarie de Litwin y Stringer $^{23}$ (Anexo 1), el cual consta de 50 preguntas que recogen información de la percepción que poseen los individuos acerca de diferentes elementos de su ambiente de trabajo. Dicho instrumento agrupa nueve dimensiones del clima organizacional: Estructura (1 a 8), Responsabilidad (9 a 15), Recompensa (16 a 21), Riesgo (22 a 26), Calidez (27 a 31), Apoyo (32 a 36), Normas (37 a 42), Conflicto (43 a 46) e Identidad (47 a 50). Las preguntas de este cuestionario son formuladas como afirmaciones positivas o negativas (Anexo 2) y cada una tiene 4 opciones a las cuales se les asigna un puntaje que va de 4 a 1 si son positivas, y de 1 a 4 si son negativas. Según el promedio obtenido, se clasifica el clima organizacional como malo $(1,00$ a 1,75$)$, regular $(1,751$ a $2,50)$, bueno $(2,51$ a 3,25$)$ o excelente $(3,251$ a 4,00$)$.

Para el análisis estadístico se creó una base de datos utilizando el programa Excel. Posteriormente estos datos fueron analizados utilizando el software estadístico InfoStat, versión 2014. Se realizó un análisis descriptivo estratificado por sexo de cada dimensión estudiada calculando media, desviación estándar y valor mínimo y máximo. Posteriormente, se compararon las medias utilizando el test $\mathrm{t}$ de Student, en las variables cuya distribución fue normal, y el test de Mann-Whitney en las distribuciones no normales. Además, se aplicó el test no paramétrico de Kruskal-Wallis para comparar las medias de cada dimensión, previa estratificación por área de trabajo. El nivel de significancia utilizado en esta investigación fue de 0,05. 


\section{RESULTADOS}

Del total de participantes en el estudio, un 57,6\% ( $n=19)$ fue de sexo femenino. En relación al área en la que desempeñaban sus funciones, se observó que un 57,6\% $(\mathrm{n}=19)$ de los trabajadores pertenecía al área de Administración y Finanzas, mientras el resto, en su mayoría, pertenecía a las áreas de Recursos Humanos y Programas y Proyectos. En la Figura 1 se presentan los promedios generales por cada dimensión evaluada del clima organizacional, destacándose que las dimensiones de recompensa $(2,21 \pm 0,52)$ y conflicto $(2,33 \pm 0,59)$ fueron las que obtuvieron el valor promedio más bajo. Los valores más altos fueron asignados a las dimensiones normas $(2,90 \pm 0,44)$ e identidad $(2,86 \pm 0,59)$.

En la Tabla 1 se presentan con detalle las estadísticas descriptivas, estratificadas por sexo, de las nueve dimensiones del clima organizacional exploradas. Se observó que para el sexo masculino la dimensión normas presentó el mayor promedio $(2,90 \pm 0,47)$, mientras que la dimensión con el valor más bajo fue recompensa $(2,25 \pm 0,58)$. En

\section{Figura 1.}

Promedios generales por dimensión.

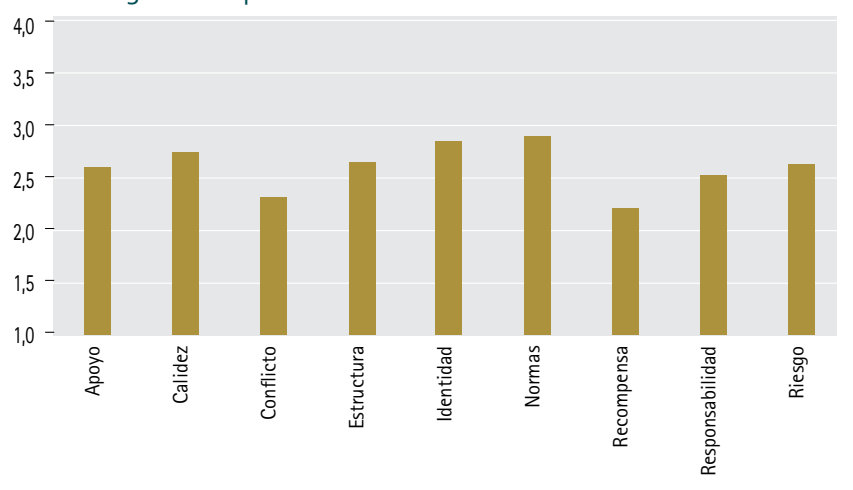

Tabla 1.

Estadisticas descriptivas separadas por sexo.

\begin{tabular}{lcccccc} 
Dimensión & \multicolumn{3}{c}{ Hombres } & \multicolumn{3}{c}{ Mujeres } \\
Estructura & Media $( \pm \mathrm{DE})$ & Mín. & Máx. & Media $( \pm \mathrm{DE})$ & Mín. & Máx. \\
Responsabilidad & $2,64 \pm 0,47$ & 1,75 & 3,38 & $2,66 \pm 0,50$ & 1,75 & 3,25 \\
Recompensa & $2,57 \pm 0,28$ & 2 & 3 & $2,50 \pm 0,21$ & 2,29 & 2,86 \\
Riesgo & $2,25 \pm 0,58$ & 1,67 & 3,67 & $2,18 \pm 0,46$ & 1,33 & 2,83 \\
Calidez & $2,68 \pm 0,54$ & 1,6 & 3,4 & $2,62 \pm 0,45$ & 1,6 & 3,2 \\
Apoyo & $2,71 \pm 0,86$ & 1 & 3,8 & $2,80 \pm 0,68$ & 1 & 3,8 \\
Normas & $2,63 \pm 0,71$ & 1,2 & 4 & $2,60 \pm 0,55$ & 1,6 & 3,6 \\
Conflicto & $2,90 \pm 0,47$ & 1,83 & 3,67 & $2,91 \pm 0,41$ & 2,17 & 3,5 \\
Identidad & $2,33 \pm 0,67$ & 1 & 4 & $2,32 \pm 0,52$ & 1,25 & 3,25 \\
& $2,86 \pm 0,51$ & 2 & 3,75 & $2,87 \pm 0,67$ & 1 & 3,75
\end{tabular}

Tabla 2.

Comparación de medias entre hombres y mujeres por dimensión.

\begin{tabular}{|c|c|c|c|c|}
\hline Dimensión & $\begin{array}{l}\text { Hombres } \\
\text { Media }\end{array}$ & $\begin{array}{l}\text { Mujeres } \\
\text { Media }\end{array}$ & $p<0.05$ & Test \\
\hline Estructura & 2,64 & 2,66 & 0,9006 & Test t-Student \\
\hline Responsabilidad & 2,57 & 2,50 & 0,3041 & Mann-Whitney \\
\hline Recompensa & 2,25 & 2,18 & 0,8419 & Mann-Whitney \\
\hline Riesgo & 2,68 & 2,62 & 0,7683 & Test t-Student \\
\hline Calidez & 2,71 & 2,80 & 0,7471 & Test t-Student \\
\hline Apoyo & 2,63 & 2,60 & 0,9103 & Test t-Student \\
\hline Normas & 2,90 & 2,91 & 0,9182 & Test t-Student \\
\hline Conflicto & 2,33 & 2,32 & 0,9825 & Test t-Student \\
\hline Identidad & 2,86 & 2,87 & 0,6871 & Mann-Whitney \\
\hline
\end{tabular}

Tabla 3.

Comparación de medias por área de trabajo.

$\begin{array}{lcccc}\text { Dimensión } & \begin{array}{c}\text { Administración } \\ \text { y Finanzas } \\ \text { Media }( \pm \mathrm{DE})\end{array} & \begin{array}{c}\text { Programas } \\ \text { y Proyectos } \\ \text { Media }( \pm \mathrm{DE})\end{array} & \begin{array}{c}\text { Recursos } \\ \text { Humanos } \\ \text { Media }( \pm \mathrm{DE})\end{array} & p<0.05 \\ \text { Estructura } & 2,64 \pm 0,48 & 2,63 \pm 0,51 & 2,75 \pm 0,48 & 0,91 \\ \text { Responsabilidad } & 2,49 \pm 0,24 & 2,57 \pm 0,27 & 2,63 \pm 0,22 & 0,48 \\ \text { Recompensa } & 2,22 \pm 0,56 & 2,13 \pm 0,51 & 2,33 \pm 0,39 & 0,67 \\ \text { Riesgo } & 2,66 \pm 0,46 & 2,67 \pm 0,65 & 2,56 \pm 0,33 & 0,61 \\ \text { Calidez } & 2,77 \pm 0,83 & 2,73 \pm 0,81 & 2,76 \pm 0,52 & 0,93 \\ \text { Apoyo } & 2,65 \pm 0,61 & 2,53 \pm 0,74 & 2,60 \pm 0,55 & 0,93 \\ \text { Normas } & 2,84 \pm 0,42 & 3,09 \pm 0,41 & 2,80 \pm 0,52 & 0,28 \\ \text { Conflicto } & 2,29 \pm 0,57 & 2,14 \pm 0,64 & 2,80 \pm 0,37 & 0,06 \\ \text { Identidad } & 2,82 \pm 0,69 & 2,83 \pm 0,41 & 3,10 \pm 0,45 & 0,59\end{array}$

el caso del sexo femenino se observó una situación similar, con un promedio de 2,91 $\pm 0,41$ para la dimensión normas y de 2,18 $\pm 0,46$ para la dimensión recompensa.

Al comparar los promedios obtenidos por hombres y mujeres en las distintas dimensiones, no se observaron diferencias significativas (Tabla 2).

En la Tabla 3 se muestran los promedios de cada dimensión estratificados por área de trabajo. El valor más bajo lo presentó el área de Programas y Proyectos en la dimensión de recompensa $(2,13 \pm 0,51)$, mientras que el más alto fue obtenido por el área de Recursos Humanos en la dimensión de identidad $(3,10 \pm 0,45)$. Al comparar los promedios no se observaron diferencias significativas.

\section{DISCUSIÓN}

En la organización estudiada se encontró que la mayoría de las dimensiones reflejaron la existencia de un buen clima organizacional; sin embargo, al abordar las dimensiones de conflicto y recompensa se observó que el clima era regular. Se constató que existían similitudes en la valoración del clima realizada tanto por hombres como por mujeres, encontrándose que en ambos casos la dimensión que manifestó un mejor clima fue la de normas y, por el contrario, que la dimensión más débil fue la de recompensa. Ninguna dimensión reflejó la existencia de un clima excelente, aunque tampoco malo, dentro de la organización.

Los hallazgos de este estudio coinciden con literatura que ha encontrado la valoración más baja del clima organizacional en la dimensión que tiene que ver con la recompensa. ${ }^{24,25,26}$ Además, se encuentra concordancia con la valoración positiva de la dimensión de identidad, la cual refleja el compromiso y grado de pertenencia hacia la organización. ${ }^{27}$ Los resultados difieren de un estudio comparativo realizado por Contreras y cols ${ }^{28}$, que encontró en una de sus poblaciones de estudio una alta valoración dada a la recompensa por el trabajo realizado.

En este estudio, las dimensiones que arrojaron un valor más bajo podrian sugerir que los miembros de la organización perciben que aun cuando aumenten su productividad laboral, la recompensa recibida por este esfuerzo seguirá siendo la misma. ${ }^{29}$ Además, en la organización podría existir un cierto grado de dificultad en las comunicaciones con la dirección. ${ }^{30}$ Por otro lado, las destacadas dimensiones de identidad y calidez podrían reflejar que los miembros de la organización se sienten vinculados a un grupo de trabajo que consideran funciona bien, y que presentan un alto grado de identificación con las metas y objetivos de la organización. ${ }^{29}$ Otra característica importante podría 
ser la existencia de buenas relaciones sociales al interior de esta empresa, junto con una clara definición de las tareas y responsabilidades asignadas. ${ }^{30}$

Como limitación de este trabajo se puede señalar la dificultad para contar con una muestra mayor debido al tamaño de la organización seleccionada.

\section{CONCLUSIÓN}

En la organización estudiada se encontró un buen clima organizacional. No se observaron diferencias significativas en la valo- ración del clima entre mujeres y hombres, coincidiendo ambos que el ámbito más débil era el de recompensa. Se sugiere la creación de instancias que permitan mejorar las comunicaciones entre la dirección y los trabajadores. Sumado a lo anterior, se hace necesario desarrollar mecanismos de recompensa que, pese a que existe una fuerte vinculación de los trabajadores con la organización y sus metas, favorezcan un ambiente de trabajo altamente motivante y productivo.

Se considera relevante el desarrollo de futuros trabajos que aborden el impacto del clima organizacional en el desempeño laboral dentro de organizaciones gestoras de cuidados en la atención primaria de salud.

\section{Anexo 1.}

Cuestionario de Estudio de Clima Organizacional (Litwin\&Stringer).

La siguiente encuesta forma parte de un estudio de Clima Organizacional que la Dirección ha decidido realizar en la organización. Debido a lo anterior nos dirigimos a usted con la finalidad de obtener una visión de cuál es el estado real de este concepto.

Desde ya, agradecemos su disposición a participar de este estudio y le pedimos que responda con la mayor sinceridad posible. Tenga la confianza de que los resultados son confidenciales y en ningún caso se utilizarán con otro fin que no sea contribuir a los resultados globales de la investigación en cuestión.

A continuación usted encontrará 50 afirmaciones acerca de cómo se desarrolla el trabajo en la Organización, frente a cada una de ellas tiene cuatro alternativas de respuestas, que son las siguientes: Totalmente de acuerdo, Relativamente de acuerdo, Relativamente en desacuerdo y Totalmente en desacuerdo. Para responder adecuadamente la siguiente encuesta, lea cuidadosamente cada pregunta marcando con una $X$ la alternativa que según su opinión describe con más exactitud la situación actual de la organización. Finalmente, le pedimos POR FAVOR NO DEJE NINGUNA INFORMACIÓN SIN CONTESTAR.
MARQUE CON UNA X:

\begin{tabular}{l|l|}
\hline ÁREA DE TRABAIO & \\
\hline ADMINISTRACIÓN Y FINANZAS & \\
\hline PROGRAMAS Y PROYECTOS & \\
\hline RECURSOS HUMANOS & \\
\hline OTRA &
\end{tabular}

\section{GÉNERO}

FEMENINO

MASCULINO

\section{NN $\quad$ AFIRMACIONES}

1 En esta organización los trabajos están bien definidos y organizados.

2 En esta organización no siempre está claro quién debe tomar las decisiones.

3 La organización se preocupa de que yo tenga claro su funcionamiento, en quienes recae la autoridad y la responsabilidad de cada trabajador.

4 En esta organización no es necesario solicitar permiso cada vez que se hace alguna cosa.

5 Las ideas nuevas no se toman mucho en cuenta, debido a que existen demasiadas reglas, detalles administrativos y trámites que cumplir.

6 A veces trabajamos en forma desorganizada y sin planificación.

7 En algunas de las labores en que me he desempeñado, no he sabido exactamente quién era mi jefe directo.

8 Quienes dirigen la organización prefieren reunir las personas más apropiadas para hacer un trabajo, aunque esto signifique cambiarlas de sus puestos habituales.

9 En esta organización cuando se encomienda una actividad hay poca confianza en la persona que asume el trabajo.

10 Quienes dirigen esta organización prefieren que quien está haciendo las cosas siga adelante con confianza, en vez de consultar con ellos.

11 En esta organización los jefes dan las indicaciones generales de lo que se debe hacer y se le deja al personal la responsabilidad e iniciativa de realizarlo.

12 Para que un trabajo quede bien, es necesario que sea hecho con audacia, responsabilidad e iniciativa.

13 Cuando se nos presentan problemas en el trabajo debemos resolverlos por nosotros mismos y no recurrir necesariamente a los jefes directos.

14 Es común en esta organización que los errores sean superados solo con disculpas.

15 Uno de los problemas que tenemos es que la gente no asume sus responsabilidades en el trabajo.

16 En esta organización los que se desempeñan mejor en su trabajo pueden llegar a ocupar los mejores puestos.

17 En esta organización existe mayor preocupación por destacar el trabajo bien hecho que aquel mal hecho.

18 En esta organización mientras mejor sea el trabajo que se haga, mejor es el reconocimiento que se recibe.

19 En esta organización existe tendencia a ser más negativo que positivo.

20 En esta organización no hay suficiente recompensa ni reconocimiento por el trabajo bien hecho.

21 En esta organización los errores son sancionados.

22 En esta organización se trabaja de forma lenta, pero segura y sin riesgos.

23 Esta organización se ha desarrollado porque se ha arriesgado cuando ha sido necesario.

24 En esta organización la toma de decisiones se hace en forma cautelosa para alcanzar los fines propuestos.

25 Las jefaturas de esta organización están dispuestas a correr riesgos ante una buena iniciativa.

26 Para que esta organización sea superior a otras, a veces hay que correr grandes riesgos.

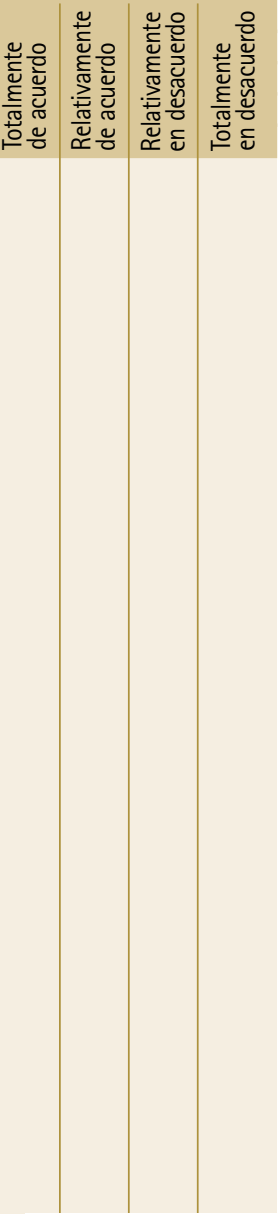


...continuación Anexo 1.

\section{№ AFIRMACIONES}

27 Entre el personal de esta organización predomina un ambiente de amistad.

28 Esta organización se caracteriza por un ambiente cómodo y relajado.

29 En esta organización cuesta mucho llegar a tener amigos.

30 En esta organización la mayoria de las personas son indiferentes hacia los demás.

31 En esta organización existen buenas relaciones humanas entre jefaturas y el resto de los funcionarios.

32 En esta organización los jefes son poco comprensivos cuando se comete un error.

33 En esta organización la jefatura se esfuerza por conocer las aspiraciones de cada funcionario.

34 En esta organización no existe mucha confianza entre superior y subordinado.

35 La jefatura de esta organización muestra interés por las personas, por sus problemas e inquietudes.

36 En esta organización cuando tengo que hacer un trabajo difícil, puedo contar con la ayuda de jefes y compañeros.

37 En esta organización se nos exige un rendimiento muy alto en nuestro trabajo.

38 Para las jefaturas de esta organización toda tarea puede ser hecha de mejor manera.

39 En esta organización las jefaturas continuamente insisten en que mejoremos nuestro trabajo individual y grupal.

40 En esta organización mejorará el rendimiento por sí solo cuando los funcionarios estén contentos.

41 En esta organización se valoran más las caracteristicas personales de los funcionarios que su rendimiento laboral.

42 En esta organización las personas parecen darle mucha importancia al hecho de hacer bien su trabajo.

43 En esta organización la mejor manera de causar buena impresión es evitar las discusiones y desacuerdos.

44 La jefatura cree que las discrepancias entre los distintos departamentos y personas pueden ser útiles a la organización.

45 En la organización se nos alienta para que digamos lo que pensamos, aunque no estemos de acuerdo con nuestros jefes.

46 En esta organización, para llegar a un acuerdo, no se toman en cuenta las distintas opiniones existentes.

47 Las personas están satisfechas de estar en esta organización.

48 Siento que pertenezco a un grupo de trabajo que funciona bien.

49 Hasta donde yo me doy cuenta, existe lealtad hacia esta organización.

50 En esta organización la mayoria de los funcionarios están más preocupados de sus propios intereses.

\section{Anexo 2.}

Clasificación de preguntas cuestionario de Litwin\&Stringer.

$\begin{array}{ll}\text { Tipo de pregunta } & \mathrm{N}^{\circ} \\ \text { Positiva } & 1,3,4,8,10,12,14,16-18,22,23,25-28,31,33,35- \\ & 40,42,44,45,47-49 \\ \text { Negativa } & 2,5,6,7,9,11,13,15,19-21,24,29,30,32,34,41,43,46,50\end{array}$


1. Noriega V. Otra aproximación al estudio del clima organizacional. Revista Infodir [en línea] 2009[citado 13 Ago2014];(9):00.Disponible en: http://bvs. sld.cu/revistas/infd/n909/infd040910.htm

2. Schneider B, Ehrhart MG, Macey WA. Perspectives on organizational climate and culture. In:Zedeck S, editor. APA handbook of industrial and organizational psychology; Vol. 1. Building and developing the organization. Washington, DC: American Psychological Association: 2011. p. 373-414.

3. Noriega V, Pria M. Instrumento para evaluar el clima organizacional en los Grupos de Control de Vectores.Rev Cubana Salud Pública. 2011; 378(2):00.

4. Guillén M. Clima organizacional en la Editorial Ciencias Médicas a partir del análisis de dos de sus dimensiones. Rev Cubana Salud Pública. 2013; 39(2):242-252.

5. Pérez I, Maldonado M, Pérez M y Bustamante S. Clima organizacional y gerencia: inductores del cambio organizacional. Investigación y Postgrado. 2006;21(2):231-248.

6. Schneider $B$, Ehrhart $M$, MaceyW. Organizational Climate and Culture. AnnuRevPsychol. 2013;64:361-88.

7. Carlfjord $S$, Andersson $A$, Nilsen $P$, Bendtsen $P$, Lindberg $M$. The importance of organizational climate and implementation strategy at the introduction of a new working tool in primary health care. J EvalClinPract. 2010;16 (6):1326-32.

8. Gershon R, Stone P, Bakken S, Larson E. Measurement of Organizational Culture and Climate in Healthcare. JNursAdm. 2004;34(1):33-40.

9. Cruvinel $E$, Richter $K$, Bastos R, Ronzani T. Screening and brief intervention for alcohol and other drug use in primary care: associations between organizational climate and practice. AddictSciClinPract. 2013; 8(1):4.

10. Poghosyan L, Nannini A, Clarke S. Organizational climate in primary care settings: implications for nurse practitioner practice. J Am Assoc Nurse Pract. 2013; 25(3):134-40.

11. Poghosyan L, Nannini A, Finkelstein S, Mason E, Shaffer J. Development and psychometric testing of the Nurse Practitioner Primary Care Organizational Climate Questionnaire. Nurs Res. 2013; 62(5):325-34.

12. Gershon R, Stone P, Zeltser M, Faucett J, MacDavitt K, Chou S. Organizational climate and nurse health outcomes in the United States: a systematic review. IndHealth. 2007; 45(5):622-36.

13. Nembhard I, Northrup V, Shaller D, Cleary P. Improving organizational climate for quality and quality of care: does membership in a collaborative help? MedCare.2012; 50:S74-82.

14. Segura A. Clima organizacional: un modo eficaz para dirigir los servicios de salud. AvEnferm. 2012; 30(1):107-113.

15. Segredo A. Clima organizacional en la gestión del cambio para el desarrollo de la organización. Rev Cubana Salud Pública. 2013; 39(2):385-393.
16. Rojas $M$, Tirado L, Pacheco $R$, Escamilla $R$, López M. El clima laboral en las unidades de hemodiálisis en México: Un estudio transversal. Nefrología. 2011; 31(1):76-83.

17. Grau $S$, Garcia M. Desgaste profesional en el personal sanitario y su relación con los factores personales y ambientales. GacSanit. 2005; 19(6): 463-470.

18. Varela 0, Puhl S, Izcurdia M. Clima laboral en el poder judicial y su incidencia en la salud mental de los profesionales que alli se desempeñan. Anulnvestig. 2012; 19(1):179-183.

19. González L, Guevara E, Morales G, Segura P, Luengo C. Relación de la satisfacción laboral con estilos de liderazgo en enfermeros de hospitales públicos, Santiago, Chile. CiencEnferm. 2013; 19(1):11-21.

20. Ramírez $M$, Lee $S$. Sindrome de Burnout entre hombres y mujeres medido por el clima y la satisfacción laboral. Polis. 2011: 10(30):431-446.

21. Cuadra A, Veloso C. Liderazgo, Clima y Satisfacción Laboral en las Organizaciones. Universum.2007; 22(2):40-56.

22. Patlán J, Flores R. Desarrollo y Validación de la Escala Multidimensional de Clima Organizacional (EMCO): Un Estudio Empírico con Profesionales de la Salud. CiencTrab.2013; 15(48):131-139.

23. Litwin G, Stringer R. Motivation and Organizational Climate. Cambridge: Harvard UniversityPress; 1968.

24. Chiang M, Salazar M, Martín M, Núñez A. Clima organizacional y satisfacción laboral. Una comparación entre hospitales públicos de alta y baja complejidad. Salud Trab. 2011; 19(1):5-16.

25. Robles-García $M$, Dierssen-Sotos $T$, Martínez-Ochoa $E$, Herrera-Carral $P$, Díaz-Mendi A, Llorca-Díaz J. Variables relacionadas con la satisfacción laboral: un estudio transversal a partir del modelo EFQM. GacSanit. 2005; 19(2):127-134.

26. Sánchez Ignacio, Airola G, Cayazzo T, Pedrals N, Rodriguez M, Villarroel L. Clima laboral en una escuela de medicina. Estudio de seguimiento. RevMéd Chile. 2009; 137(11):1427-1436.

27. Llapa-Rodríguez $E_{1}$ Trevizan $M$, Shinyashiki $T$, Mendes A. Compromiso organizacional y profesional del equipo de salud. EnfermGlob. 2009; (17):0-0.

28. Contreras $F$, Barbosa $D$, Juárez $F$, Uribe $A$, Mejia C. Estilos de liderazgo, clima organizacional y riesgos psicosociales en entidades del sector salud: un estudio comparativo. Act Colom Psicol. 2009; 12(2):13-26.

29. Chiang M, Salazar M, Martin M, Nuñez A. Clima organizacional y satisfacción laboral en un establecimiento de salud estatal: hospital tipo 1. Theoria.2007; 16(2): 61-76.

30. Roch G, Dubois C, Clarke S. Organizational climate and hospital nurses' caring practices: a mixed-methods study. Res NursHealth. 2014; 37(3):229-40. 